\title{
Theoretical estimations of future polarization observations for exoplanery searches with gravitational microlensing
}

\author{
Alexander F. Zakharov* \\ Institute of Theoretical and Experimental Physics, Moscow, 117259, Russia \\ Bogoliubov Laboratory of Theoretical Physics, JINR, Dubna, 141980 Russia \\ North Carolina Central University, Durham, NC 27707, USA \\ National Research Nuclear University MEPhI (Moscow Engineering Physics Institute), 115409, \\ Moscow, Russia \\ E-mail: zakharov@itep.ru
}

Gabriele Ingrosso, Francesco De Paolis, Achille A. Nucita, Francesco Strafella

Dipartimento di Matematica e Fisica "Ennio De Giorgi”, Università del Salento, CP 193,

I-73100 Lecce, Italy

E-mail: ingrosso, depaolis, nucita, strafelladle.infn.it

\section{Sebastiano Calchi Novati}

Dipartimento di Fisica “E.R. Caianiello", Università di Salerno, I-84081 Baronissi (SA), Italy

Istituto Internazionale per gli Alti Studi Scientifici (IIASS), Vietri Sul Mare (SA), Italy

E-mail: novatidsa.infn.it

\section{Philippe Jetzer}

Institute for Theoretical Physics, University of Zürich, Winterthurerstrasse 190, CH-8057,

Zürich, Switzerland

E-mail: ietzerdphysik.uzh.ch

\begin{abstract}
There are different methods for finding exoplanets such as radial spectral shifts, astrometrical measurements, transits, timing etc. Gravitational microlensing (including pixel-lensing) is among the most promising techniques with the potentiality of detecting Earth-like planets at distances about a few astronomical units from their host star or near the so-called snow line with a temperature in the range $0-100^{\circ} \mathrm{C}$ on a solid surface of an exoplanet. We emphasize the importance of polarization measurements which can help to resolve degeneracies in theoretical models. In particular, the polarization angle could give additional information about the relative position of the lens with respect to the source.
\end{abstract}

XXII International Baldin Seminar on High Energy Physics Problems 15-20 September, 2014

JINR, Dubna, Russia

\footnotetext{
* Speaker.
} 


\section{Introduction}

Exoplanet search are one of the hottest topic of modern astronomy because here we have an intersection of many different branches of science including not only astronomy and astrophysics but also astrobiology including abundance and origin of life [四].

Already before the discovery of exoplanets Mao and Paczynski showed how efficient is gravitational microlensing as a tool to search for extrasolar planets, including the low mass ones, even at relatively large distances from their host stars [వ]. Later on, observations and simulations gave the opportunity to confirm the robustness of these conclusions. Exoplanets near the snow line may be also detected with this technique as it was shown, for instance, in Fig. 8 presented in [B]. Moreover, in contrast with conventional methods, such as transits and Doppler shift measurements, gravitational microlensing ${ }^{1}$ gives a chance to find exoplanets not only in the Milky Way

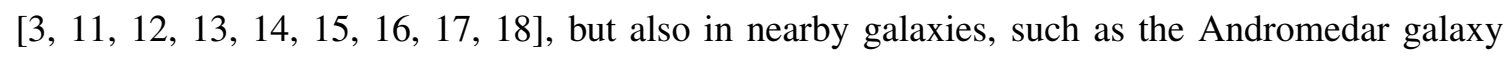
[ㅁ, [20], so pixel-lensing towards M31 provides an efficient tool to search for exoplanets and indeed an exoplanet might have been already discovered in the PA-N2-99 event [एप, 201]. Since source stars for pixel-lensing towards M31 are basically red giants (and therefore, their typical diameters are comparable to Einstein diameters and the caustic sizes) one has to take into account the source finiteness effect [D2]. In the case of relatively small size sources, the probability to have features due to binary lens (or planet around star) in the light curves is also small since it is proportional to the caustic area. Giant star sources have large angular sizes and relatively higher probability to touch caustics [ए]].

In the paper we point out an importance of polarization observations for microlensing event candidates to support (or reject) microlensing model and resolve degeneracies of binary (exoplanetary) microlens models.

\section{Exoplanet Searches with Gravitational Microlensing}

Since the existence of planets around lens stars leads to the violation of circular symmetry of lens system and, as a result, to the formation of fold and cusp type caustics [23], 24, 25], one can detect extra peaks in the microlensing light curve due to caustic crossing by the star source as a result of its proper motion.

A list of exoplanets detected with microlensing searches toward the Galactic bulge is given in

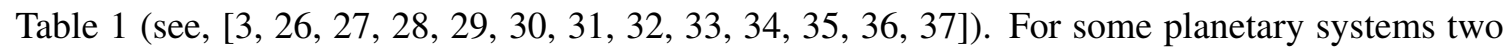
probable regions for the planet-to-star distance are given due to the planet and star-lens parameter degeneracy [28, [88], see rows 9, 14, 17 in Table $\mathbb{W}$. Reports about these discoveries were published

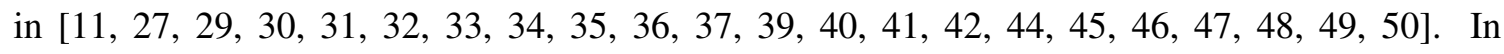
these searches we have a continuous transition from massive exoplanets to brown dwarfs, since an analysis of the anomalous microlensing event, MOA-2010-BLG-073 has been done [ 5$]$ ], where the primary of the lens is an M-dwarf with $M_{L 1}=0.16 \pm 0.03 M_{\odot}$ while the companion has $M_{L 2}=$

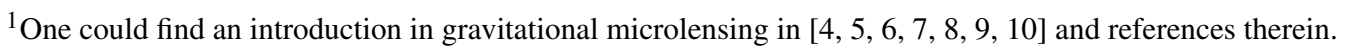


Table 1: Exoplanets discovered with microlensing. 27 exoplanets have been found in 25 systems, in particular, there are two exoplanets in OGLE-2006-BLG-109L (lines 5,6) and there are two exoplanets in OGLE2012-BLG-0026 (lines 18,19), there is an Earth mass exoplanet in microlensing event OGLE-2013-BLG0341 (line 25), where the exoplanet is located near one star in binary stellar system.

\begin{tabular}{|c|c|c|c|c|}
\hline$\#$ & Star Mass $\left(M_{\odot}\right)$ & Planet Mass & Star-planet Separation (AU) & Reference \\
\hline 1 & $0.63_{-0.09}^{+0.07}$ & $830_{-190}^{+250} M_{\oplus}$ & $4.3_{-0.8}^{+2.5}$ & [B9],[26] \\
\hline 2 & $0.46 \pm 0.04$ & $(1100 \pm 100) M_{\oplus}$ & $(3.6 \pm 0.2)$ & [40], [29] \\
\hline 3 & $0.22_{-0.11}^{+0.21}$ & $5.5_{-2.7}^{+5.5} M_{\oplus}$ & $2.6_{-0.6}^{+1.5}$ & [ㅁ] ] \\
\hline 4 & $0.49_{-0.29}^{+0.23}$ & $13_{-8.0}^{+6.0} M_{\oplus}$ & $2.7_{-1.4}^{+1.7}$ & [4]] \\
\hline 5 & $0.51_{-0.04}^{+0.05}$ & $(230 \pm 19) M_{\oplus}$ & $(2.3 \pm 0.5)$ & [42] \\
\hline 6 & $0.51_{-0.04}^{+0.05}$ & $(86 \pm 7) M_{\oplus}$ & $4.5_{-1.0}^{+2.1}$ & [42] \\
\hline 7 & $0.64_{-0.26}^{+0.21}$ & $20_{-8}^{+7} M_{\oplus}$ & $3.3_{-0.8}^{+1.4}$ & [4] \\
\hline 8 & $0.084_{-0.012}^{+0.015}$ & $3.2_{-1.8}^{+5.2} M_{\oplus}$ & $0.66_{-0.14}^{+0.19}$ & [27] \\
\hline 9 & $0.30_{-0.12}^{+0.19}$ & $260.54_{-104.85}^{+165.22} M_{\oplus}$ & $0.72_{-0.16}^{+0.38} / 6.5_{-1.2}^{+3.2}$ & [BO] $]$ \\
\hline 10 & $0.67 \pm 0.14$ & $28_{-23}^{+58} M_{\oplus}$ & $1.4_{-0.3}^{+0.7}$ & [44] \\
\hline 11 & $0.38_{-0.18}^{+0.34}$ & $50_{-24}^{+44} M_{\oplus}$ & $2.4_{-0.6}^{+1.2}$ & [45] \\
\hline 12 & $0.19_{-0.12}^{+0.30}$ & $2.6_{-1.6}^{+4.2} M_{\mathrm{J}}$ & $1.8_{-0.7}^{+0.9}$ & [46] \\
\hline 13 & $0.56 \pm 0.09$ & $10.4 \pm 1.7 M_{\oplus}$ & $3.2_{-0.5}^{+1.9}$ & [47] \\
\hline 14 & $0.44_{-0.17}^{+0.27}$ & $2.4_{-0.6}^{+1.2} M_{\mathrm{J}}$ & $1.0 \pm 0.1 / 3.5 \pm 0.5$ & [48] \\
\hline 15 & $0.67_{-0.13}^{+0.33}$ & $1.5_{-0.3}^{+0.8} M_{\mathrm{J}}$ & $2_{-1}^{+3}$ & [49] \\
\hline 16 & $0.75_{-0.41}^{+0.33}$ & $3.7 \pm 2.1 M_{\mathrm{J}}$ & $8.3_{-2.7}^{+4.5}$ & [50] \\
\hline 17 & $0.26 \pm 0.11$ & $0.53 \pm 0.21 M_{\mathrm{J}}$ & $2.72 \pm 0.75 / 1.50 \pm 0.50$ & [B]] \\
\hline 18 & $0.82 \pm 0.13$ & $0.11 \pm 0.02 M_{\mathrm{J}}$ & $3.82 \pm 0.30$ & [B2] \\
\hline 19 & $0.82 \pm 0.13$ & $0.53 \pm 0.21 M_{\mathrm{J}}$ & $4.63 \pm 0.37$ & [B2] \\
\hline 20 & $0.022 \pm 0.002$ & $1.88 \pm 0.19 M_{\mathrm{J}}$ & $0.88 \pm 0.03$ & [B3] \\
\hline 21 & $0.44 \pm 0.07$ & $2.73 \pm 0.43 M_{\mathrm{J}}$ & $3.45 \pm 0.26$ & {$[34$, B5] $]$} \\
\hline 22 & $0.11 \pm 0.01$ & $9.2 \pm 2.2 M_{\oplus}$ & $0.92 \pm 0.16$ & [B6] \\
\hline 23 & $0.025 \pm 0.001$ & $9.4 \pm 0.5 M_{\mathrm{J}}$ & $0.19 \pm 0.01$ & [B]] \\
\hline 24 & $0.018 \pm 0.001$ & $7.2 \pm 0.5 M_{\mathrm{J}}$ & $0.31 \pm 0.01$ & [B7] \\
\hline 25 & $0.1-0.15$ & $\sim 2 M_{\oplus}$ & $\sim 0.8$ & [52] \\
\hline 26 & $0.23 \pm 0.07$ & $1.0 \pm 0.3 M_{\mathrm{J}}$ & 2 & [53] \\
\hline 27 & $0.39_{0.19}^{+0.45}$ & $11.6_{-5.6}^{+13.4} M_{\mathrm{J}}$ & $4.3_{-1.22}^{+1.5}$ & [54] \\
\hline
\end{tabular}

$11.0 \pm 2.0 M_{\mathrm{J}}^{2}$, at a perpendicular distance around $1.21 \pm 0.16 \mathrm{AU}$ from the host star, so the low mass component of the system is near a boundary between planets and brown dwarfs.

It is remarkable that the first exoplanet has been discovered by the MOA-I collaboration with

\footnotetext{
${ }^{2}$ According to the definition of a "planet" done by the working group of the International Astronomical Union on February 28, 2003 has the following statement: "... Objects with true masses below the limiting mass for thermonuclear fusion of deuterium (currently calculated to be 13 Jupiter masses for objects of solar metallicity) that orbit stars or stellar remnants are "planets" (no matter how they formed).."
} 


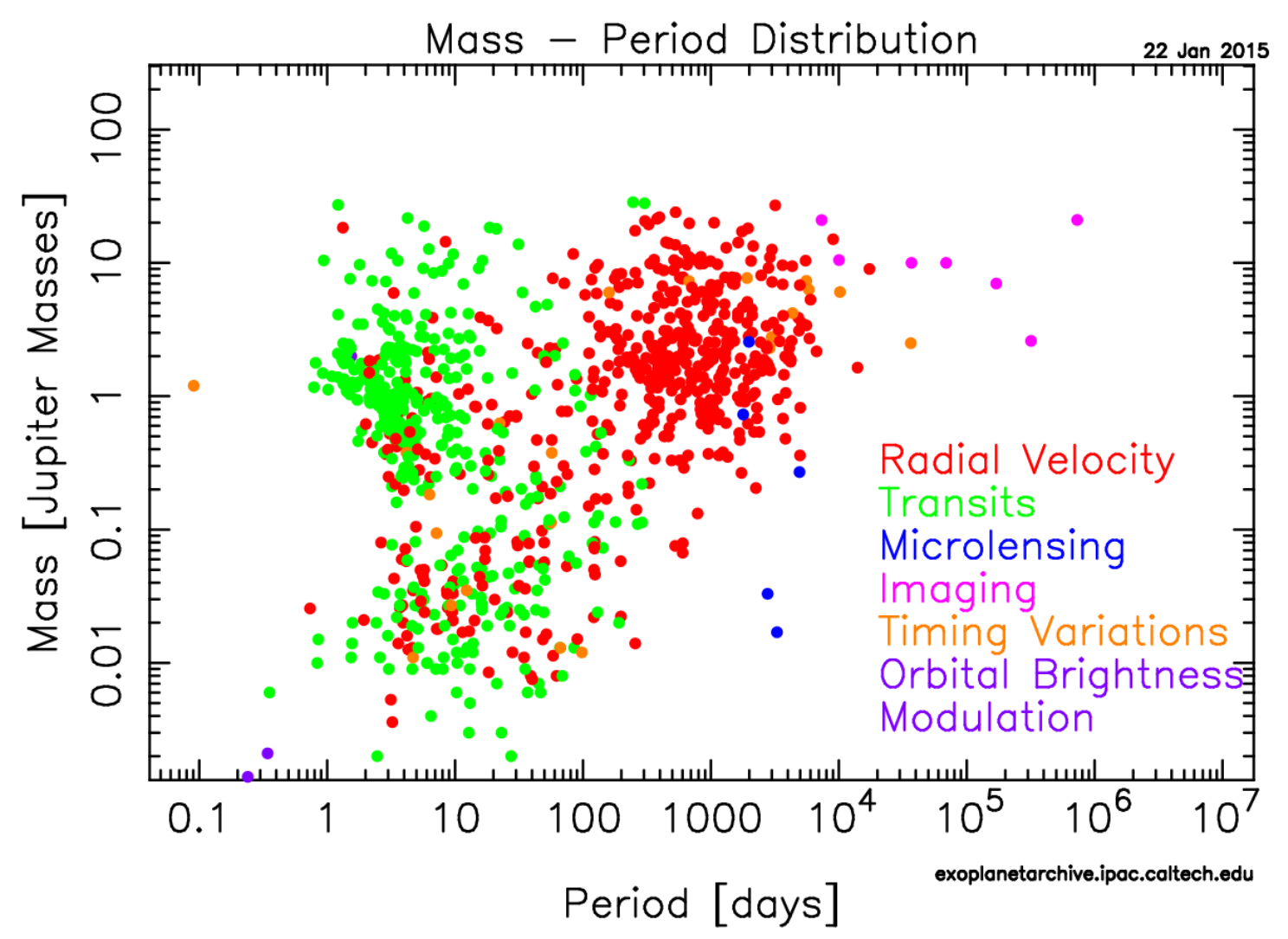

Figure 1: Mass - period distribution for all exoplanets found with different techniques until January 22, 2015, see http://exoplanetarchive.ipac.caltech.edu/exoplanetplots/.

only a $0.6 \mathrm{~m}$ telescope [28, $3 \mathrm{Bg}]$. This microlensing event was also detected by the OGLE collaboration, but the MOA observations with a larger field of view CCD, made about 5 exposures per night for each of their fields. This was an important advantage and shows that even observations with modest facilities (around 1 meter telescope size and even smaller) can give a crucial contribution in such discoveries. Until now four super-Earth exoplanets (with masses about $10 M_{\oplus}$ ) have been discovered by microlensing (see Table Wand Fig. W), showing that this technique is very efficient in detecting Earth mass exoplanets at a few AU from their host stars, since a significant fraction of all exoplanets discovered with different techniques and located in the region near the so-called snow line (or the habitable zone) found with gravitational microlensing. Some of these exoplanets are among the lighest exoplanets see lines 3 and 8 in Table $\mathbb{W}$. For comparison, Doppler shift measurements help to detect an Earth-mass planet orbiting our neighbor star a Centauri B. The planet has an orbital period of 3.236 days and is about $0.04 \mathrm{AU}$ from the star [55]. Recently, a sub-Mercury size exoplanet Kepler-37b has been discovered with a transit technique [56]. It means that the existence of cool rocky planets is a common phenomenon in the Universe [], [5], [8]. Moreover, recently, in [5] it was claimed that around $17 \%$ of stars host Jupiter-mass planets $\left(0.3-10 M_{\mathrm{J}}\right)$, cool Neptunes $\left(10-30 M_{\oplus}\right)$ and super-Earths $\left(10-30 M_{\oplus}\right)$ have relative abundances per star in the Milky Way such as 52\% and 62\%, respectively.

Analysis of Kepler space telescope data also shows that a the most stars have to have exoplan- 


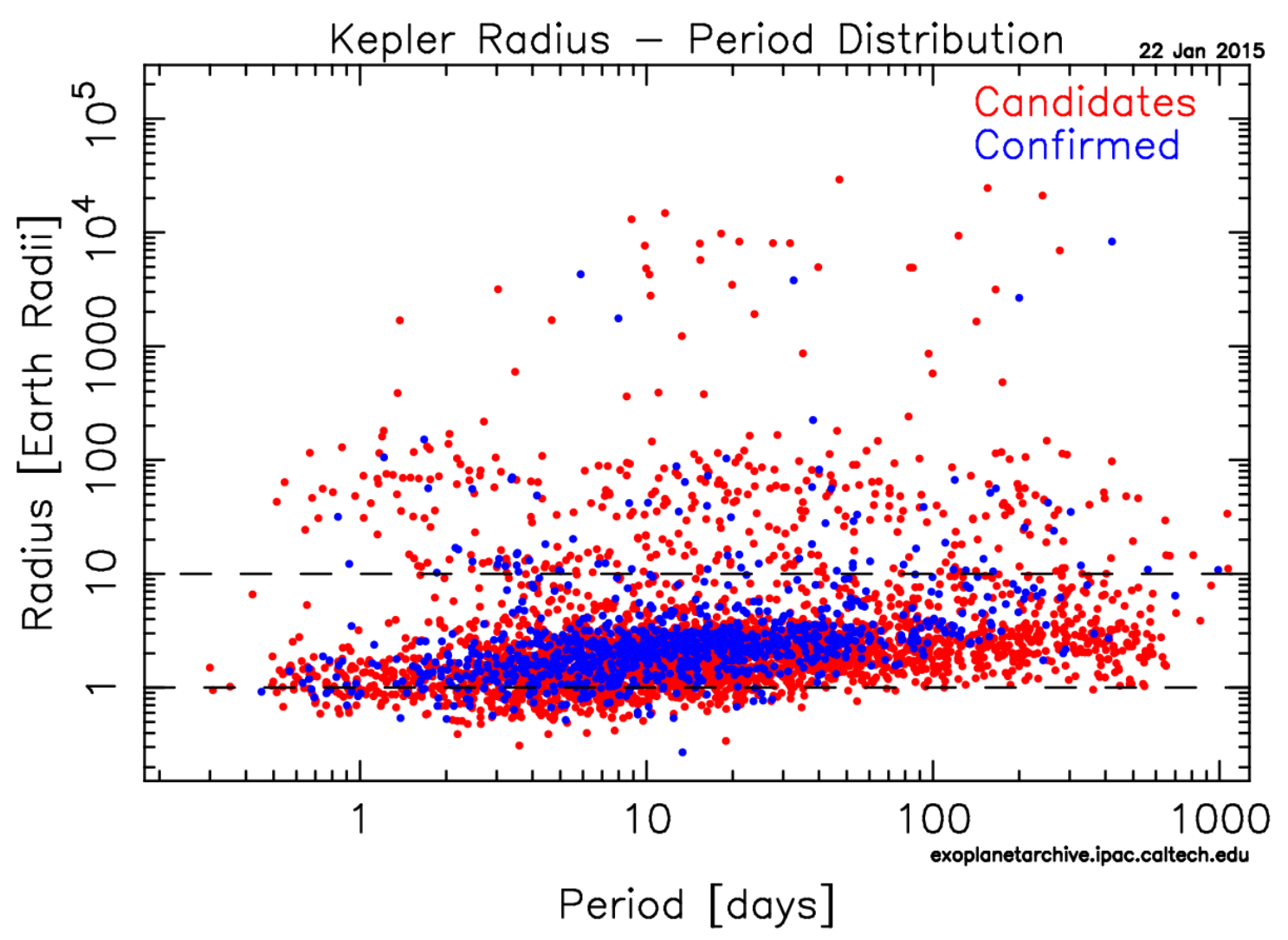

Figure 2: Mass - period distribution for all exoplanet (candidates and confirmed) events found with transit technique. Distribution shows results of data analysis until January 22, 2015, see http://exoplanetarchive.ipac.caltech.edu/exoplanetplots/.

ets [6]]. An analysis of transits with the Kepler telescopes led to conclusions about an existence of a number of exoplanets with small radius showing that not only exoplanet existence is a wide spread phenomenon, but also small size exoplanets with relatively long periods are rather abundant (see Fig. 1 or more precisely, one finds $5.7_{-2.2}^{+1.7} \%$ of Sun-like stars harbor an Earth-size planet with orbital periods of 200-400 days [ 6 ]. In January 2015 more than 1000 exoplanet event candidates have been confirmed. ${ }^{3}$ The Kepler mission finished its observations in 2013 but data analysis is still going on.

Clearly, that if angular sizes of source stars are comparable with corresponding angular impact parameters and Einstein - Chwolson angles then light curves for such sources are different from the standard Paczyński light curve and gravitational lensing could be colorful since one has limb darkening and color distribution for extended background stars [ [62, 63, 64, 65], 66]. The extended source effects in gravitational microlensing enable studying the stellar atmospheres through their limb-darkening profiles and by modelling their microlensed spectra, see [67, 68, 69, 미, 므, 미, 미, [74, [75, [06, [7] and references therein for details.

Pixel-lensing towards M31 may provide an efficient tool to search for exoplanets in that galaxy

\footnotetext{
${ }^{3} \mathrm{http} / / /$ exoplanetarchive.ipac.caltech.edu/docs/exonews_archive.html\#15Jan2015.
} 


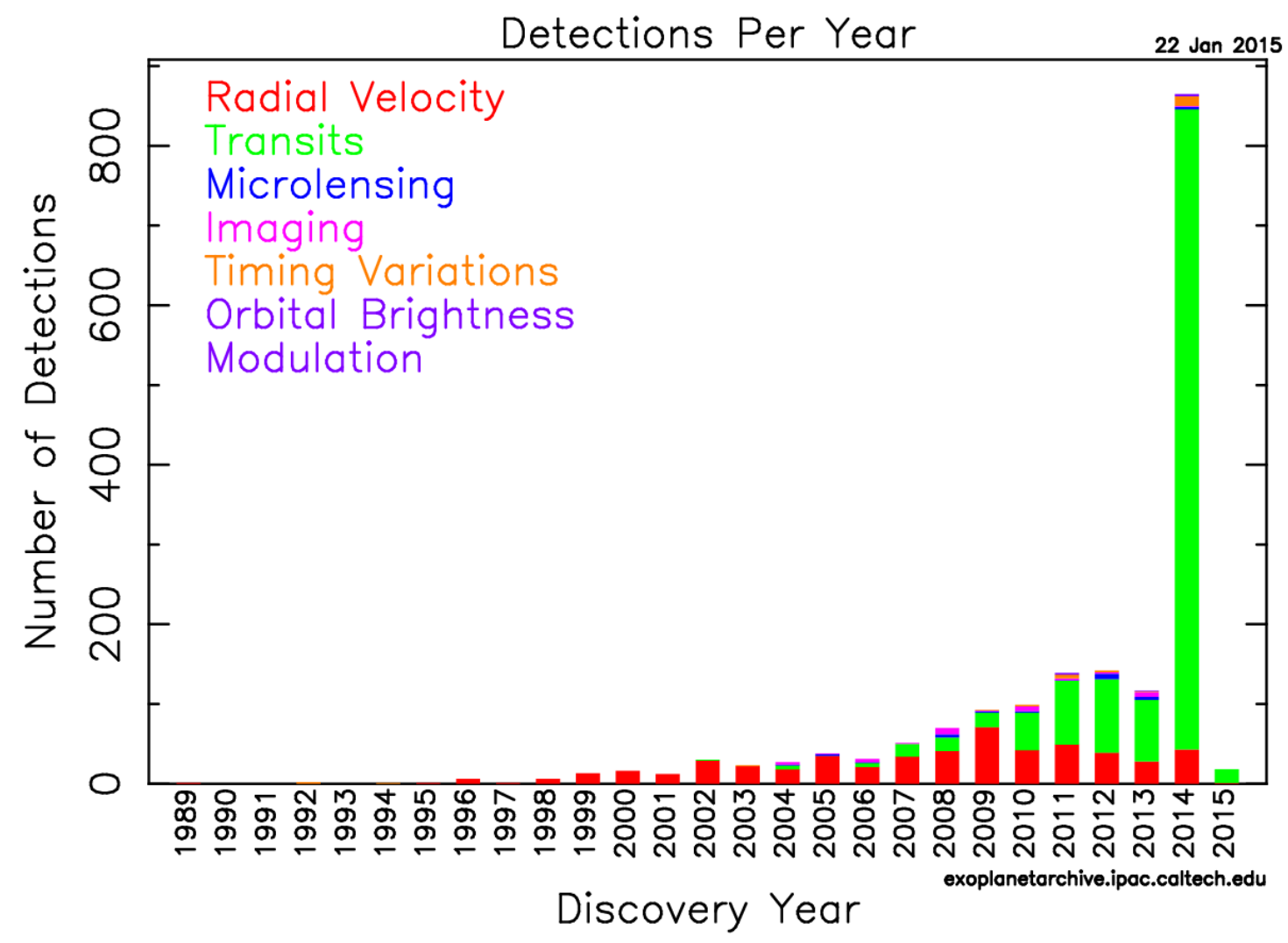

Figure 3: Diagram of exoplanet discovery evolution. The most of exoplanets have been found with the transit technique and Kepler observations in 2014.

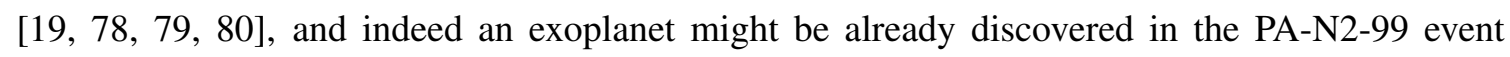
[एव]. Since source stars for pixel-lensing towards M31 are basically red giants (and therefore, their typical diameters are comparable to Einstein diameters and the caustic sizes) one has to take into

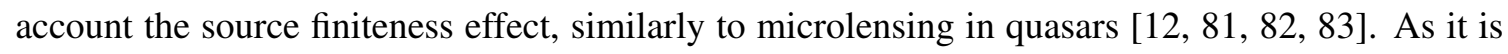
well known the amplifications for a finite source and for a point-like source are different because there is a gradient of amplification in respect of a source area. If the source size is rather small, the probability to produce features of binary lens (or planet around star) is proportional to the caustic area. However, giant stars have large angular sizes and relatively higher probability to

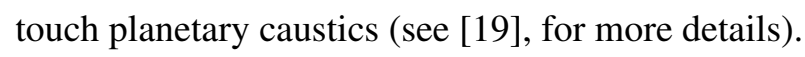

\section{Polarization curves for microlens systems with exoplanets}

For extended sources, the importance of polarization measurements was pointed out for pointlike lens in [84] and for binary lens in [85] (see also, [86]). For point-like lens polarization could reach $0.1 \%$ while for binary lens it could reach a few percent since the magnification gradient is much greater near caustics. It has been shown that polarization measurements could resolve degeneracies in theoretical models of microlensing events [85]. Calculations of polarization curves for microlensing events with features in the light curves induced by the presence of an exoplanet and 


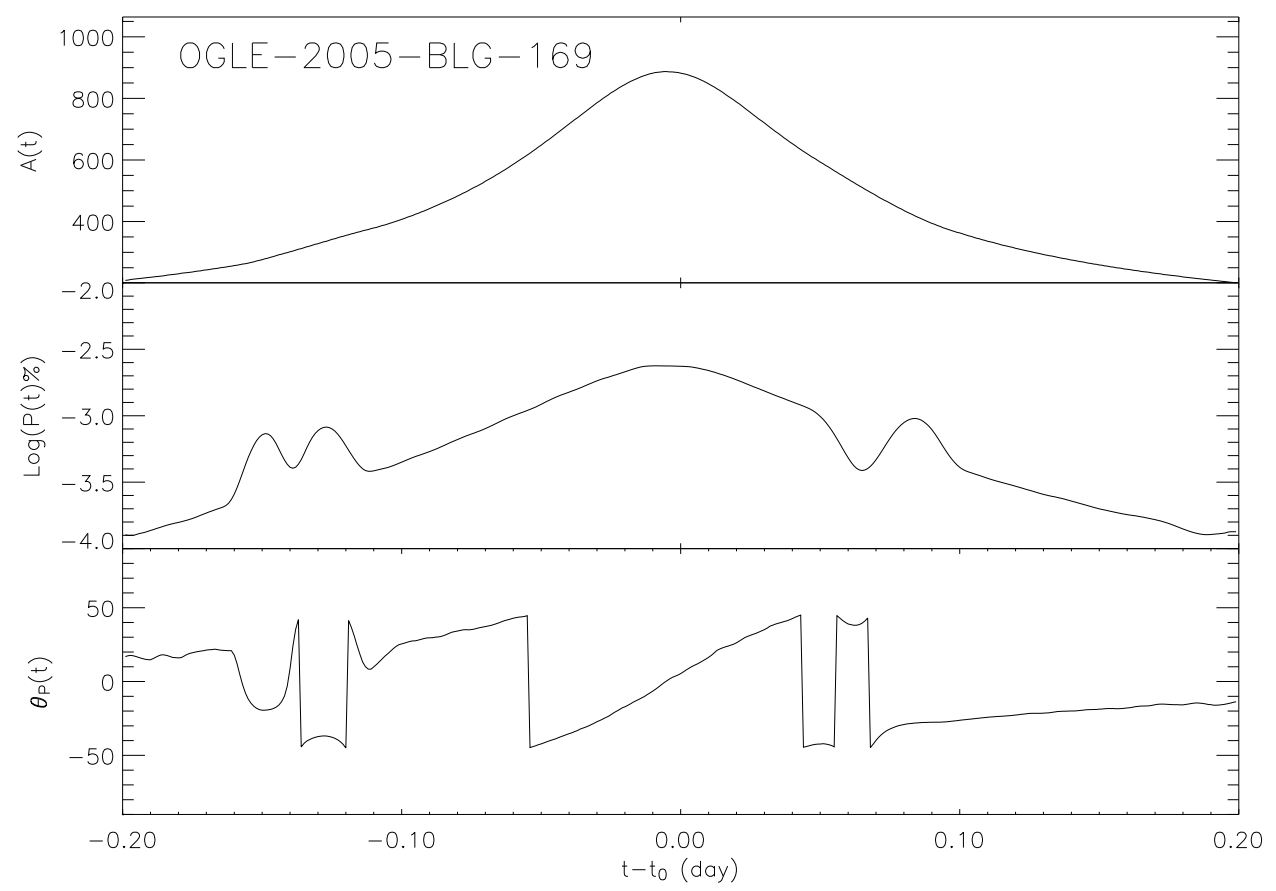

Figure 4: Light curve (top panel), polarization curve (middle panel) and polarization angle (bottom panel) for the OGLE-2005-BLG-169 event.

observed towards the Galactic bulge have been done [87, 88, 89, 001, 0]]. We use simple polarization and limb darkening models developed by [92], however, improved models are also developed taking into account radiative transfer in spectral lines, see for instance simulation results developed for Sun [03]. Here we emphasize that measurements of then polarization angle could give additional information about the gravitational microlensing model. ${ }^{4}$ If polarization measurements are possible, in principle, one could measure polarization as a function of direction for an orientation of polarimeter and an instant for microlensing event. If a polarimeter is fixed one has an additional function of time to explain observational data, but if a polarimeter could be rotated, polarization is an additional function of direction at each instant. Such an information could help to resolve degeneracies and confirm (or disprove) microlensing models for observed phenomena. For instance, for a point-like lens the direction for the maximal polarization at the instant when an amplification is also maximal (which is perpendicular to the line connecting star and lens) may allow to infer the direction of lens proper motion, thus allowing to eventually pinpoint the lens in following observations. Even in the case of binary lens, the orientation of polarization vector corresponds to the orientation of the fold caustic (or more correctly to the tangent vector to the fold caustic at the intersection point with the path of source), provided the source size is small enough.

\footnotetext{
${ }^{4}$ We call polarization angle the angle which corresponds to a direction with the maximal polarization.
} 
In Fig. 田, the light curve, the polarization curve and the polarization angle are shown for the OGLE-2005-BLG-169 event, where a binary system formed by a main sequence star with mass $M_{\odot} \sim 0.5 M_{\odot}$ and a Neptune-like exoplanet with mass about $13 M_{\oplus}$ is expected from the light curve analysis [4]]. The event parameters are $t_{E}=42.27$ days, $u_{0}=1.24 \times 10^{-3}, b=1.0198$, $q=8.6 \times 10^{-5}, \alpha=117.0 \mathrm{deg}, \rho_{*}=4.4 \times 10^{-4}$, where $t_{E}, u_{0}, b, q, \alpha, \rho_{*}$ are the Einstein time, the impact parameter, the projected distance of the exoplanet to the host star, the binary component mass ratio, the angle formed by the source trajectory and the separation vector between the lenses, and the source star size, respectively (all distances are given in $R_{E}$ units). The effect of the source transiting the caustic (see [4] is clearly visible both in the polarization curve (see the middle panel in Fig. (1) and in the flip of the polarization angle (see the bottom panel). We would like to stress that the high peak magnification $(A \simeq 800)$ of the OGLE-2005-BLG-169 event leading to $I$-magnitude of the source about 13 mag at the maximum gives the opportunity to measure the polarization signal for such kind of events by using present available facilities. In this case, polarization measurements might give additional information about the caustic structure, thus potentially allowing to distinguish among different models of exoplanetary systems. Recently, [04] found that a variable giant star source mimics exoplanetary signatures in the MOA-2010-BLG-523S event. In this respect, we emphasize that polarization measurements may be helpful in distinguishing exoplanetary features from other effects in the light curves.

The polarization curve and the polarization angle for the MOA-2008-BLG-310Lb event is shown in Fig. [1. For this event it was expected the existence of a sub-Saturn exoplanet with mass $m=74 \pm 17 M_{\oplus}$ [44]]. The event parameters are $t_{E}=11.14$ days, $u_{0}=3 . \times 10^{-3}, b=1.085$, $q=3.31 \times 10^{-4}, \alpha=69.33 \mathrm{deg}, \rho_{*}=4.93 \times 10^{-3}$. In particular, the event is characterized by large finite source effect since $\rho_{*} / u_{0}>1$, leading to polarization features similar to those of single lens events. Nevertheless, in this case we can see the variability in the polarization signal that arises when the source touches the first fold caustic at $t_{1} \simeq t_{0}-0.07$ days, the source enters the primary lens at $t_{2} \simeq t_{0}-t_{E} \sqrt{\rho_{*}^{2}-u_{0}^{2}}$ days, the source exits the primary lens at $t_{3} \simeq t_{0}+t_{E} \sqrt{\rho_{*}^{2}-u_{0}^{2}}$ days and touches the second fold caustic $t_{4} \simeq t_{0}+0.09$ days (see also Fig. 4 in paper by [4]]).

\section{Conclusions}

Now there are campaigns of wide field observations by Optical Gravitational Lensing Experiment (OGLE) [05] and Microlensing Observations in Astrophysics (MOA) [06] and a couple of follow-up observations, including MicroFUN ${ }^{5}$ and PLANET. ${ }^{6}$ It is important to note that small size (even less than one meter diameter) telescopes acting in follow-up campaigns contributed in discoveries of light Earth-like exoplanets and it is a nice illustration that a great science can be done with modest facilities. As it was shown in [87] polarization measurements are very perspective to remove uncertainties in exoplanet system determination and they give an extra proof for a conventional gravitational microlens model with suspected exoplanets. Moreover, an orientation of polarization angle near the maximum of polarizations (and light) curves gives information on direction of proper motion in respect to gravitational microlens system which could include exoplanet.

\footnotetext{
${ }^{5}$ http://www.astronomy.ohio-state.edu/ microfun/microfun.html.

${ }^{6}$ http://planet.iap.fr/.
} 


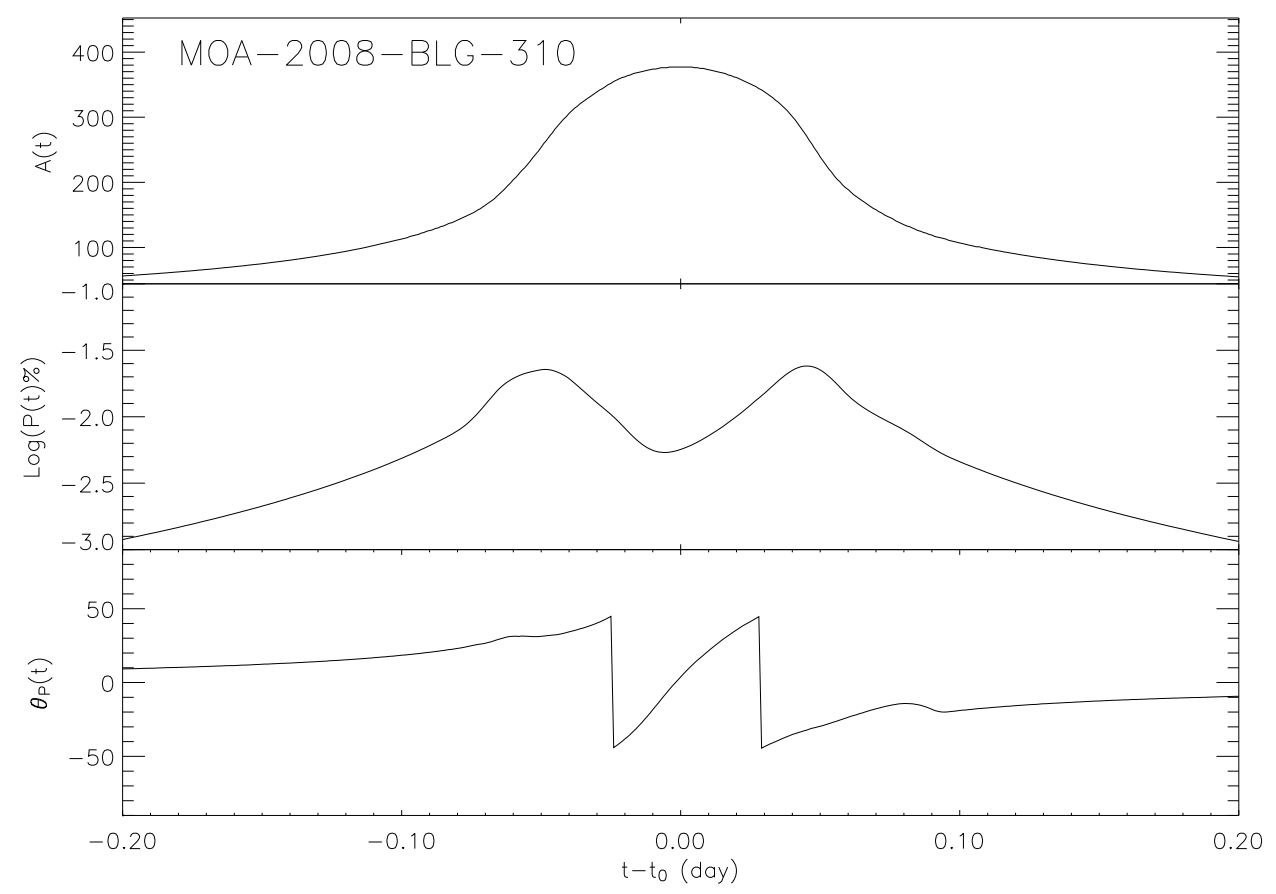

Figure 5: Light curve (top panel), polarization curve (middle panel) and polarization angle (bottom panel) for the OGLE-2008-BLG-310 event.

Such an information could be important for possible further observations of the gravitational lens system in future.

Astronomers discovered a significant number of exoplanets with observations of gravitational microlensing toward the Galactic bulge due to an effective synergy of wide field monitoring by OGLE collaboration and follow up observations with even small telescopes distributed over the globe. These follow up observations are possible since the Early Warning System (EWS) of the OGLE group designed for detection of microlensing events in progress reported about microlensing event candidates and these candidates are carefully monitored with other telescopes located in different time zones. It gives an opportunity to use telescopes with facilities for polarimetric observations. To increase efficiency of exoplanet detection with pixel lensing similar early warning system is needed for pixel lensing observations because it give an opportunity for follow up observations of suspected cases and for polarimetric observations with available facilities. It means that observational facilities for monitoring, follow up and polarimetric observations are ready, but effective software for online analysis of pixel lensing observations has to be developed. An existence of EWS for pixel lensing give an opportunity to get continuous series of observational data for the most pixel lensing event candidates and avoid gaps in light curves for suspected events. For example, the observed light curve for very interesting PA-99-N2 event has 20 day gap in an important place for gravitational lens model reconstruction, but the light curve showed signatures 
of star and exoplanet in Andromeda galaxy [ㅍ] , so it was extremely important to get continuous series of observations for such events.

\section{Acknowledgments}

AFZ thanks organizers of the XXII International Baldin Seminar on High Energy Physics Problems to this contribution and for a financial support. AFZ acknowledges also a partial support of the NSF (HRD-0833184) and NASA (NNX09AV07A) grants at NCCU (Durham, NC, USA).

\section{References}

[1] M. Perryman, The Exoplanet Handbook, Cambridge University Press, 2014.

[2] S. Mao, B. Paczynski, Gravitational microlensing by double stars and planetary systems, ApJ 374 (1991) L37.

[3] S. Mao, Astrophysical applications of gravitational microlensing, RAA 12 (2012) 947.

[4] A.V. Byalko, Focusing of Radiation by a Gravitational Field, Soviet Astronomy, 13 (1970) 784.

[5] B. Paczynski, Gravitational microlensing by the galactic halo, ApJ 304 (1986) 1.

[6] B. Paczynski, Gravitational microlensing by the galactic halo, ARA\&A 34 (1996) 419.

[7] E. Roulet, S. Mollerach, Microlensing, Physics Reports, 279 (1997) 67.

[8] A.F. Zakharov, M.V. Sazhin, Gravitational microlensing, Physics - Uspekhi 41 (1998) 945.

[9] A.F. Zakharov, Gravitational microlensing: results and perspectives, Publications of the Astronomical Observatory of Belgrade, 75 (2003) 27.

[10] A. F. Zakharov, Gravitational Microlensing: Results and Perspectives in Brief, Physics of Particles and Nuclei, 39 (2008) 1176.

[11] J.-P. Beaulieu, D. P. Bennett, P. Fouqué et al., Discovery of a cool planet of 5.5 Earth masses through gravitational microlensing, Nature 439 (2006) 437.

[12] A.F. Zakharov, Gravitational lensing: From micro to nano, New Astron. Rev. 53 (2009) 202.

[13] M. Dominik, Studying planet populations by gravitational microlensing, Gen. Rel. Grav. 42 (2010) 2075.

[14] A.F. Zakharov, S. Calchi Novati, F. De Paolis, et al., Exoplanet searches with gravitational microlensing, Mem. della Soc. Astron. Ital. Suppl. 15 (2010) 114.

[15] A.F. Zakharov, Exoplanet search with gravitational microlensing, Physics - Uspekhi 54 (2011) 1077.

[16] J.T.Wright, B. S. Gaudi, Exoplanet Detection Methods, in Planets, Stars and Stellar Systems, edited by T.D. Oswalt, L.M. French, P. Kalas, Springer Science+Business Media Dordrecht, 2013, p. 489, [arXiv:1210.2471v2[astro-ph.EP]].

[17] B. S.Gaudi, Microlensing Surveys for Exoplanets, Annual Rev. Astron. \& Astrophys. 50 (2012) 411.

[18] J.-P. Beaulieu, P. Tisserand, V. Batista, Space based microlensing planet searches, Hot Planets and Cool Stars, Edited by R. Saglia, Garching, EPJ Web of Conferences 47 (2013) 15001-1-8.

[19] G. Ingrosso, S. Calchi Novati, F. de Paolis, et al., Pixel lensing as a way to detect extrasolar planets in M31, Mon. Not. R. Astron. Soc. 399 (2009) 219. 
[20] G. Ingrosso, S. Calchi Novati, F. de Paolis, et al., Search for exoplanets in M31 with pixel-lensing and the PA-99-N2 event revisited, Gen. Rel. Grav. 43 (2011) 1047.

[21] J. H. An, N. W., Evans, E. Kerins et al., The Anomaly in the Candidate Microlensing Event PA-99-N2, ApJ 601 (2004) 845.

[22] O. Pejcha, D. Heyrovský, Extended-Source Effect and Chromaticity in Two-Point-Mass Microlensing, ApJ 690, (2009) 1772.

[23] P. Schneider, J. Ehlers, E.E. Falco, Gravitational Lenses, Springer, Berlin, 1992.

[24] A.F. Zakharov, On the magnification of gravitational lens images near cusps, Astron. \& Astrophys. 293, (1995) 1.

[25] A.O. Petters, H. Levine, J. Wambsganss, Singularity Theory and Gravitational Lensing, Birkhäuser, Boston, 2001.

[26] D. P. Bennett, J. Anderson, I.A. Bond et al., Identification of the OGLE-2003-BLG-235/MOA-2003-BLG-53 Planetary Host Star, ApJ Lett. 647 (2006) L171.

[27] D.P. Bennett, I.A. Bond, A. Udalski et al., A Low-Mass Planet with a Possible Sub-Stellar-Mass Host in Microlensing Event MOA-2007-BLG-192, ApJ 684 (2008) 663.

[28] D.P. Bennett, Detection of Extrasolar Planets by Gravitational Microlensing, [arXiv:0902.1761v1[astro-ph.EP]].

[29] S. Dong, A. Gould, A. Udalski et al., OGLE-2005-BLG-071Lb, the Most Massive M Dwarf Planetary Companion? ApJ 695 (2009) 970.

[30] S. Dong, I.A. Bond, A. Gould et al., Microlensing Event MOA-2007-BLG-400: Exhuming the Buried Signature of a Cool, Jovian-Mass Planet, ApJ 698 (2009) 1826.

[31] N. Kains, R.A. Street, J.-Y. Choi et al., A giant planet beyond the snow line in microlensing event OGLE-2011-BLG-0251, Astron. \& Astrophys. 50 (2013) 411.

[32] C. Han, A. Udalski, J.-Y. Choi, et al. The Second Multiple-planet System Discovered by Microlensing: OGLE-2012-BLG-0026Lb, cÜA Pair of Jovian Planets beyond the Snow Line, ApJ 762 (2013) L28.

[33] C. Han, Y.K. Jung, A. Udalski et al., Microlensing Discovery of a Tight, Low-mass-ratio Planetary-mass Object around an Old Field Brown Dwarf, ApJ 778 (2013) 38 (6pp.).

[34] Y. Tsapras, J.-Y., Choi, R. Street et al., A Super-Jupiter orbiting a late-type star: A refined analysis of microlensing event OGLE-2012-BLG-0406, ApJ 782 (2014) 48 (9 pp.);

[arxiv:1310.2428v1[astro-ph.EP]].

[35] R. Poleski, A. Udalski, S. Dong et al., Super-massive planets around late-type stars - the case of OGLE-2012-BLG-0406Lb,ApJ 782 (2014) 47 (8 pp.); [arxiv:1307.4084v1[astro-ph.EP]].

[36] Furusawa, K., Udalski, A., Sumi, T., et al., MOA-2010-BLG-328Lb: a sub-Neptune orbiting very late M dwarf? ApJ 779 (2014) 91 (12 pp.); [arXiv:1309.7714v2 [astro-ph.EP]].

[37] J.-Y. Choi, C. Han, A. Udalski et al., Microlensing Discovery of a Population of Very Tight, Very Low Mass Binary Brown Dwarfs, ApJ 768 (2013) 129 (7pp.).

[38] M. Dominik, The binary gravitational lens and its extreme cases, Astron. \& Astrophys. 349 (1999) 108. 
[39] I.A. Bond, A. Udalski, M. Jaroszynski et al., OGLE 2003-BLG-235/MOA 2003-BLG-53: A Planetary Microlensing Event, ApJ Lett. 606 (2004) L155.

[40] A. Udalski, M. Jaroszynski, B. Paczyński et al., A Jovian-Mass Planet in Microlensing Event OGLE-2005-BLG-071, ApJ Lett. 628 (2005) L109.

[41] A. Gould, A. Udalski, D. An et al., Microlens OGLE-2005-BLG-169 Implies That Cool Neptune-like Planets Are Common, ApJ 644 (2006) L37.

[42] B.S. Gaudi, D. P. Bennett, A. Udalski et al., Discovery of a Jupiter/Saturn Analog with Gravitational Microlensing, Science 319 (2008) 927.

[43] T. Sumi, D.P. Bennett, I.A. Bond et al., A cold Neptune-mass planet OGLE-2007-BLG-368Lb: cold Neptunes are common, ApJ 710 (2010) 1641.

[44] J. Janczak, A. Fukui, S. Dong et al., Sub-Saturn Planet MOA-2008-BLG-310Lb: Likely to be in the Galactic Bulge, ApJ 711 (2010) 731.

[45] N. Miyake, T. Sumi, S. Dong et al., A sub-Saturn mass planet MOA-2009-BLG-319Lb, ApJ 728 (2011) 120 (10pp.).

[46] V. Batista, A. Gould, S. Dieters et al., MOA-2009-BLG-387Lb: a massive planet orbiting an M dwarf, Astron. \& Astrophys., 529 (2011) A102 (16pp.).

[47] Y. Muraki et al., Discovery and Mass Measurements of a Cold, 10 Earth Mass Planet and Its Host Star, ApJ 741 (2011) 22 (15pp.).

[48] J. C. Yee, Y. Shvartzvald, A. Gal-Yam et al., MOA-2011-BLG-293Lb: A Test of Pure Survey Microlensing Planet Detections, ApJ 755 (2012) 102 (14pp.).

[49] E. Bachelet, I.-G. Shin, C. Han et al., MOA 2010-BLG-477Lb: Constraining the Mass of a Microlensing Planet from Microlensing Parallax, Orbital Motion, and Detection of Blended Light, ApJ 754 (2012) 73 (17pp.).

[50] D.P. Bennett, T. Sumi, I.A. Bond et al., Planetary and Other Short Binary Microlensing Events from the MOA Short-event Analysis, ApJ 757 (2012) 119 (17pp.).

[51] R. A. Street, J.-Y. Choi, Y. Tsapras et al., MOA-2010-BLG-073L: An M-dwarf with a Substellar Companion at the Planet/Brown Dwarf Boundary, ApJ 763 (2013) 63 (13pp.).

[52] A. Gould, A. Udalski, I.-G. Shin et al. A Terrestrial Planet in a $\sim 1$ AU Orbit Around One Member of $a \sim 15$ AU Binary, Science 345 (2014) 46.

[53] J. Skowron, I.-G. Shinu, A. Udalski, OGLE-2011-BLG-0265Lb: a Jovian Microlensing Planet Orbiting an M Dwarf, [arXiv:1410.8252v1 [astro-ph.EP]].

[54] Y. Shvartzvald, D. Maoz, S. Kaspi et al., MOA-2011-BLG-322Lb: a "second generation survey" microlensing planet, Mon. Not. R. Astron. Soc. 439 (2014) 604.

[55] X. Dumusque, F. Pepe, C. Lovis et al., An Earth-mass planet orbiting a Centauri B, Nature 491 (2012) 207.

[56] Th. Barclay, J.F. Rowe, J. J. Lissauer et al., A sub-Mercury-sized exoplanet, Nature 494 (2013) 452.

[57] M. Dominik, Stochastic distributions of lens and source properties for observed galactic microlensing events, Mon. Not. R. Astron. Soc. 367 (2006) 669.

[58] M. Dominik, K. Horne, M.F. Bode, The first cool rocky/icy exoplanet, Astron. \& Geophys. 367 (2006) 3.25 . 
[59] J.-P. Cassan, D. Kubas, J-P. Beaulieu et al., One or more bound planets per Milky Way star from microlensing observations, Nature 481 (2012) 167.

[60] F. Fressin, G. Torres, D. Charbonneau et al., The False Positive Rate of Kepler and the Occurrence of Planets, ApJ 766 (2013), 81 (20pp.).

[61] E. A. Petigura, A. W. Howard, and G.W. Marcy, Prevalence of Earth-size planets orbiting Sun-like stars, PNAS 110 (2013) 19273.

[62] H. J. Witt, S. Mao, Can lensed stars be regarded as pointlike for microlensing by MACHOs? ApJ 430 (1994) 505.

[63] H.J. Witt, The Effect of the Stellar Size on Microlensing at the Baade Window, ApJ 449 (1995) 42.

[64] M.B. Bogdanov, A. M. Cherepashchuk, The "chromatic” effect in gravitational microlensing of stars, Astron. Lett. 40 (1995) 505.

[65] M.B. Bogdanov, A.M. Cherepashchuk, Gravitational microlensing of stars of nonzero angular size, Astron. Rep. 40 (1995) 713.

[66] M.B. Bogdanov, A. M. Cherepashchuk, Reconstruction of the brightness distribution over a stellar disk from observations of gravitational microlensing, Astron. Rep. 40 (1996) 717.

[67] A. Loeb, D. Sasselov, Removing Degeneracy of Microlensing Light Curves through Narrowband Photometry of Giants, ApJ 449 (1995) L33.

[68] D. Sasselov, Opportunities for stellar surface imaging via gravitational microlensing, in proceedings Cool stars; stellar systems; and the Sun, Astronomical Society of the Pacific Conference Series, 109, Proceedings of the 9th Cambridge Workshop, San Francisco, Astronomical Society of the Pacific (ASP), edited by R. Pallavicini and A.K. Dupree, p.541 (1996).

[69] C. Alcock, W.H. Allen, R.A.N. Allsman et al., MACHO Alert 95-30: First Real-Time Observation of Extended Source Effects in Gravitational Microlensing, ApJ 491 (1997) 436.

[70] D. Sasselov, Surface Imaging by Microlensing, in Cool stars; stellar systems; and the Sun, ASP Conf. Ser. 154, The Tenth Cambridge Workshop on Cool Stars, Stellar Systems and the Sun, edited by R.A. Donahue, J.A. Bookbinder, p.383 (1998).

[71] D. Valls-Gabaud, Chromatic and spectroscopic signatures of microlensing events as a tool for the gravitational imaging of stars, Mon. Not. R. Astron. Soc. 294 (1998).

[72] D. Heyrovský, D. Sasselov, A. Loeb, Probing Red Giant Atmospheres with Gravitational Microlensing, ApJ 546 (2000) 406.

[73] H.M. Bryce, M.A. Hendry, D. Valls-Gabaud, Gravitational microlensing as a test of stellar model atmospheres, Astron. \& Astrophys. 388 (2002) L1.

[74] J.-P. Cassan, J.-P. Beaulieu, S. Brillant et al., Probing the atmosphere of the bulge G5III star

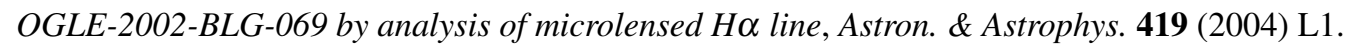

[75] J.-P. Cassan, J.-P. Beaulieu, P. Fouqué et al., OGLE 2004-BLG-254: a K3 III Galactic bulge giant spatially resolved by a single microlens, Astron. \& Astrophys. 460 (2006) 277.

[76] C. Thurl, P.D. Sackett, P. H., Hauschildt, Resolving stellar atmospheres. I. The H $\alpha$ line and comparisons to microlensing observations, Astron. \& Astrophys. 455 (2006) 315.

[77] M. Zub, A. Cassan, D. Heyrovský et al. Limb-darkening measurements for a cool red giant in microlensing event OGLE 2004-BLG-482, Astron. \& Astrophys. 525 (2011) A15-12, 2011. 
[78] S.-J. Chung, D. Kim, M.J. Darnley et al., The Possibility of Detecting Planets in the Andromeda Galaxy, ApJ 650 (2006) 432.

[79] Kim, D., Chung, S.-J., Darnley, M. J., et al., Detection of M31 Binaries via High-Cadence Pixel-lensing Surveys,ApJ 666 (2007) 236.

[80] A. F. Zakharov, G. Ingrosso, F. De Paolis et al., Exoplanetary searches with gravitational microlensing, Publications of Astronomical Observatory of Belgrade 92 (2013) 65.

[81] E. Agol \& J. Krolik, Imaging a Quasar Accretion Disk with Microlensing, ApJ 524 (1999) 49.

[82] L.Č. Popović, P. Jovanović, E. Mediavilla et al., A Study of the Correlation between the Amplification of the Fe $K_{\alpha}$ Line and the X-Ray Continuum of Quasars due to Microlensing, ApJ 637 (2006) 620.

[83] P. Jovanović, A.F. Zakharov, L. Č. Popović, T. Petrović, Microlensing of the X-ray, UV and optical emission regions of quasars: simulations of the time-scales and amplitude variations of microlensing events, Mon. Not. R. Astron. Soc. 386 (2008) 397.

[84] M.B. Bogdanov, A. M. Cherepashchuk, M.V. Sazhin, M. V., Microlensing and Polarization, Astrophys. \& Space Science 235 (1996) 219.

[85] E. Agol, Polarization during binary microlensing, Mon. Not. R. Astron. Soc. 279 (1996) 571.

[86] R. Ignace, E. Bjorkman \& H.M. Bryce, The polarization signature from microlensing of circumstellar envelopes in caustic crossing events, Mon. Not. R. Astron. Soc. 366 (2006) 92.

[87] G. Ingrosso, S. Calchi Novati, F. de Paolis et al., Polarization in microlensing events towards the Galactic bulge, Mon. Not. R. Astron. Soc. 426 (2012) 1496.

[88] G. Ingrosso, F. De Paolis, A. A. Nucita et al., Polarization in binary microlensing events, Physica Scripta 89 (2014) 084001, (6pp); arXiv: 1310.5866v1[astro-ph.SR].

[89] A. F. Zakharov, G.Ingrosso, F. De Paolis et al., Exoplanetary searches with gravitational microlensing: polarization issues, Advances in Space Research 54 (2014) 1319.

[90] A. F. Zakharov, G.Ingrosso, F. De Paolis et al., Exoplanet Searches in the Habitable Zone with Gravitational Microlensing, in proceedings of the International Astronomical Union, Volume 8 Symposium S293 (Formation, Detection, and Characterization of Extrasolar Habitable Planets), Cambridge University Press, p. 362014.

[91] A.F. Zakharov, G. Ingrosso, F. De Paolis et al., Exoplanet searches with gravitational microlensing: polarization aspects, in proceeding of the XV Advanced Research Workshop on High Energy Spin Physics (DSPIN-13), edited by A.V. Efremov and S.V. Goloskokov, p. 155, JINR, Dubna, 2014.

[92] S. Chandrasekhar, Radiative Transfer, Dover Publications, New York, 1960.

[93] J.O. Stenflo, Polarization at the Extreme Limb of the Sun and the Role of Eclipse Observations, in Proceedings of the International Symposium on Solar Physics and Solar Eclipses (SPSE 2006) held at Waw an Namos, Libya, 27-29 March 2006, edited by R. Ramelli, O. Shalabiea, I. Saleh, J.O. Stenflo, p.1 (2006)

[94] A. Gould, J.C. Yee, I. A. Bond et al., MOA-2010-BLG-523: "Failed Planet" = RS CVn Star, ApJ 763 (2013) 141 (11pp.).

[95] A. Udalski, The Optical Gravitational Lensing Experiment. Real Time Data Analysis Systems in the OGLE-III Survey, Acta Astron. 53 (2003) 291.

[96] I.A. Bond, F. Abe, R.J. Dodd et al., Real-time difference imaging analysis of MOA Galactic bulge observations during 2000, Mon. Not. R. Astron. Soc. 327 (2001) 868. 